\title{
Las pymes bogotanas y sus procesos de internacionalización. Un primer balance.
}

\author{
PEDRO PABLO CARDOZO \\ ANDRÉS CHAVARRO \\ CARLOS ARIEL RAMÍREZ
}

\section{RESUMEN}

Este artículo realiza un primer balance sobre las pymes en general y las de base tecnológica en particular, en cuanto a su internacionalización en el caso de Bogotá en años recientes (2000-2007). Revisa el estado actual de la pyme en Colombia y luego discute la bibliografía relacionada con este tema.

PALABRAS CLAVE

Internacionalización, pymes, base tecnológica.

\section{INTRODUCCIÓN}

Este artículo pretende hacer una primera introspección al estudio de las pymes y su internacionalización en el caso de Bogotá en años recientes (del 2000 en adelante). Este trabajo es el fruto de una investigación realizada por los autores sobre "Prácticas de Internacionalización Adoptadas por algunas Pymes de Base Tecnológica en Bogotá" auspiciada por el Politécnico Grancolombiano entre los años 2005 y 2006.

El plan del artículo es el siguiente: en la primera parte se establecen algunas generalidades de la pyme en Colombia para luego, en la segunda sección, abordar las condiciones y características de las pymes en Bogotá. Para ilustrar la situación de las empresas de base tecnológica en Bogotá, en esta sección hay una referencia específica a algunos sectores industriales. La tercera parte consiste en una revisión de la bibliografía teórico-práctica sobre internacionalización en Bogotá. El artículo termina con una reflexión sobre el tema central.

\section{PERFIL GENERAL DE LA PYME EN COLOMBIA}

\section{Algunas definiciones básicas}

Siguiendo las definiciones propuestas por la ley 590 de 2000 o Ley de Pymes (modificada por la ley 905 de 2003) se asumen las siguientes definiciones: 


\section{Mediana empresa}

a. Planta de personal entre cincuenta y uno (51) y doscientos (200) trabajadores; o

b. Activos totales por valor entre cinco mil uno (5.001) y quince mil (30.000) salarios mínimos mensuales legales vigentes.

\section{Pequeña empresa}

a. Planta de personal entre once (11) y cincuenta (50) trabajadores; o

b. Activos totales por valor entre quinientos uno (501) y menos de cinco mil (5.000) salarios mínimos mensuales legales vigentes.

\section{Microempresa}

a. Planta de personal no superior a los diez (10) trabajadores; o

b. Activos totales por valor inferior a quinientos uno (501) salarios mínimos mensuales legales vigentes.

\section{Cuatro características fundamentales}

a. Su actividad principal se dirige a hacer algún desarrollo tecnológico.

b. Tienen una clara intención de hacer innovación ya sea radical o incremental y pueden centrarse en el producto o en el proceso.

c. Por su misma naturaleza tienen una intensa actividad de I+D.

d El proceso de I+D es realizado por personal científico y técnico altamente calificado.

En algunos contextos internacionales, como convocatorias a proyectos de incubación ${ }^{1}$ se definen las empresas de base tecnológica como aquellas que además de cumplir con las anteriores características, exploten derechos de propiedad intelectual como parte clave de su negocio y que su propiedad industrial esté protegida.

Para algunos estudiosos en internacionalización (Jones 1999, Fillis, 2001) las firmas de alta tecnología se caracterizan porque trabajan en mercados internacionales altamente cambiantes, donde crean su principal ventaja basada en la habilidad para desarrollar y explotar las innovaciones desarrolladas, lo que implica segmentar mercados y ser capaces de adaptarse rápidamente al cambio (Jones 1999).

\section{Pedro Pablo Castro Cardoso}

Biólogo Marino de la Universidad Jorge Tadeo Lozano, Oficial Naval Mercante adscrito al Cuerpo de Oficiales Logísticos de la Academia Naval Almirante Cristóbal Colon, Maestría en Auditoria y Planificación Ambiental Empresarial del Instituto de Estudios Ecológicos de Málaga (España), Maestría en Comercio Internacional del Instituto de Estudios Bursátiles de la Bolsa de Madrid - Universidad Complutense (Madrid - España). Observador Internacional de los protocolos de La Jolla (CA), Consultant- Trainner de Orbimage, Corp; Gerente para Centroamérica y el Caribe de Nautical Transoceanic Ltda. Consultor en Sistemas de Gestión Integrados de Matcom Consultores S.A. Profesor Investigador del grupo de Desarrollo Sostenible del Politécnico Grancolombiano, profesor catedrático y Director de Proyectos de Grado de la Maestría en Gestión Ambiental de la Pontificia Universidad Javeriana: profesor de la Maestría en Administración de Empresa de la Universidad Externado de Colombia. Candidato a PhD de la Escuela de Administración de Empresas de la Universidad de Hull (Inglaterra).

Para contactar al autor: ppccardoso2@gmail.com

\section{Andrés Chavarro}

Economista Universidad Nacional de Colombia. Especialista en Derecho Público- Administrativo de la misma universidad. Estudiante de Maestría en Ciencias Económicas de la Universidad Nacional. Diplomado en Docencia Universitaria del Politécnico Grancolombiano. Diplomado en Competencias para la Docencia Universitaria del Politécnico Grancolombiano. Diplomado en investigación del Politécnico Grancolombiano en asociación con la ACAC. Miembro del grupo de investigación en sostenibilidad del Politécnico Grancolombiano registrado ante Colciencias. Docente investigador del Politécnico Grancolombiano.

Para contactar al autor: achavarr@poligran.edu.co

\section{Carlos Ariel Ramírez}

Economista Master en Ciencias Económicas de la Universidad Nacional de Colombia. Docente de tiempo completo del área económica del Politécnico Grancolombiano. Catedrático de la Universidad Central. Diplomado en Docencia Universitaria del Politécnico Grancolombiano. Diplomado en Tutoría en Ambientes Virtuales de Aprendizaje de la Universidad Autónoma de Bucaramanga. Miembro del grupo en sostenibilidad del Politécnico Grancolombiano registrado ante Colciencias.

Para contactar al autor: carlosar@poligran.edu.co

1 Asociación para el Desarrollo Regional de Cantabria. Programa Regional de Acciones Innovadoras, 2004. 
Para Fillis (2001) muchas de las pequeñas empresas de alta tecnología nacen globales (born global); esto es, tienen una visión global, operan en mercados internacionales desde el principio y pertenecen a redes globales especializadas funcionalmente. Sus directores son visionarios emprendedores, quienes ven el mundo como un mercado sin fronteras y usualmente poseen la habilidad de reconocer y explotar oportunidades tecnológicas. En general, estas empresas no sólo se internacionalizan en los eslabones usuales de la cadena de valor (distribución-comercialización), sino que además, frecuentemente las áreas de I+D y producción son ejecutadas con socios internacionales; por ejemplo, por medio de convenios de cooperación o alianzas estratégicas.

\section{Algunos datos de referencia}

De acuerdo con la encuesta nacional del Dane sobre micro establecimientos, para el año 2000 se identificaron en Colombia 967.345 empresas cuyo número de empleados no excede las diez personas ${ }^{2}$.

Sin embargo, existe un problema de informalidad que lleva a cuestionar el tamaño de la cifra. Según la Superintendencia Bancaria, solamente doce mil empresas de las consideradas pymes han sido sujetos de crédito tradicional canalizado por bancos en los últimos siete años en Colombia. Esto quiere decir que la inmensa mayoría de pequeños negocios no están registrados, no piden crédito, prácticamente son invisibles, auque existan ${ }^{3}$.

Sin embargo ese sector genera en la ciudad el 63\% del empleo, el 45\% de la producción manufacturera, el 40\% de los salarios y el $37 \%$ del valor agregado ${ }^{4}$.

En cuanto al tipo de negocio, de la totalidad de los microestablecimientos en 2000, el 58\% funcionaban en el comercio, $30 \%$ en el área de servicios y $12,5 \%$ en la industrial. De estos datos, se concluye que las pymes se ubican mayoritariamente en el sector terciario de la economía, lo cual está relacionado positivamente con el aporte al valor agregado que hacen y con su participación en el PIB (11,5\% en 2002) ${ }^{5}$.

En función de la ubicación geográfica, las empresas, sin importar el tamaño, tienen tres principales plazas de localización: Bogotá con 161.217, Antioquia con 74.417 y Valle del Cauca, con 67.931 ${ }^{6}$.

Al grupo de las pymes prestan su apoyo el Banco Interamericano de Desarrollo (BID), Fundes, Finamérica, Fundación Carvajal y algunos bancos. Estima el diario Portafolio que son cuatrocientos mil (400.000) los micro-créditos girados al sector.

Según Portafolio, basado en datos de Expopymes en 2003, las pymes exportadoras vendieron entre $110 \mathrm{y}$ 120 millones de dólares en los mercados externos.

Los principales destinos fueron Estados Unidos, con una participación de 26\%, Ecuador con un 15\%, y Venezuela con 13\%. La categoría "otros países", representa el 28\%. Es de destacar el crecimiento de las pymes de vocación exportadora. Según Expopymes, se pasó de 314 empresas en 1999 a 606 en julio de 2003, lo que implica un crecimiento de $93 \%$ en tres años y medio ${ }^{7}$. Consecuentemente, las exportaciones de las pymes pasaron de 48 millones de dólares en 1999 a 61 millones (precios FOB), teniendo como pico exportador el año 2001, con 110 millones.

\section{La oferta de apoyo para las pymes en Colombia}

Continuando con el papel del sector privado y no gubernamental en el apoyo a las pymes, surge una figura de reciente creación: las incubadoras de empresas. Generalmente son organizaciones sin ánimo de lucro que tienen un doble papel. En primer lugar, las incubadoras proponen ideas y planes de negocio, lo que significa que promueve la creación de empresa. En segundo lugar, sirven como instrumentos de promoción del desarrollo tecnológico de las pymes; esto es, ofrecen servicios de tecnología que las empresas pequeñas y medianas no obtendrían por sí mismas ${ }^{8}$.

2 Suplemento especial diario Portafolio. "Pymes: La empresa día a día, manejo de situaciones cotidianas y su entorno". Bogotá: Casa Editorial El Tiempo y Empresa de Teléfonos de Bogotá. Fascículo 1. Bogotá (Colombia

3 Ibíd.

4 Ibíd. p. 2.

5 Ibíd. p. 19.

6 Ibíd. p. 21.

7 Ibíd. p. 146.

8 Ibíd. p. 5. 
Otro frente del tema es lo referente a la política de fomento diseñada por el gobierno. Toda la política de promoción está basada en la ley 590 de 2000, conocida como la ley de pymes. Las estrategias contenidas en la ley involucran varios tópicos. El primero, consiste en generar una estructura institucional encabezada por el Consejo Superior de Pequeña y Mediana Empresa, conformado, entre otros, por seis ministros, el Director de Planeación Nacional, el Presidente de ACOPI y el Presidente de Confecámaras, entre otros. El consejo tiene por funciones, entre otras:

1. Contribuir a la definición, formulación y ejecución de programas de promoción de las pymes, con énfasis en los referidos al acceso a los mercados de bienes y servicios, formación de capital humano, modernización y desarrollo tecnológico y mayor acceso a los mercados financieros institucionales.

2. Estimular el desarrollo de las organizaciones empresariales, la asociatividad y las alianzas estratégicas entre las entidades públicas y privadas de apoyo a este sector.

3."Fomentar la conformación y operación de Consejos Departamentales para el Desarrollo Productivo, así como la formulación de políticas departamentales de desarrollo de las pymes, en pro de la competitividad y estimulando cadenas de valor a niveles subregional y sectorial dentro del marco del Plan Nacional de Desarrollo ${ }^{9}$.

Se observa que el Consejo posee poder de acción en virtud de la presencia de varios ministros. Las funciones que aquí se resaltan tienden a fortalecer lo que se puede llamar competitividad; o sea, la generación de un entorno favorable y condiciones internas para el sostenimiento, mejoramiento y crecimiento de las pymes.

La estrategia de apoyo incluye también un trato especial del gobierno al sector, que se materializa a través de cuatro acciones:

1. Atención a las mipymes por parte de las entidades estatales. Se refiere a que cada oficina gubernamental que pertenece al Consejo y aquellas que están relacionadas, pero pertenecen al Consejo (Instituto de Fomento Industrial, el Fondo Nacional de Garantías, el Sena, Colciencias, Bancoldex y Proexport), deberán abrir una dependencia especializada para la atención de este tipo de empresas.
2. Registro único de las mipymes con la idea de reducir los trámites ante el Estado.

3. Concurrencia de las mipymes a los mercados de bienes y servicios que crea el Estado, para que a la hora de la contratación por parte del mismo, los funcionarios públicos permitan la participación de las pymes en igualdad de condiciones. En algunas ocasiones se ordena que: "Las entidades públicas del orden nacional, departamental y municipal, preferirán en condiciones de igual precio, calidad y capacidad de suministros y servicio a las mipymes nacionales".

4. Políticas y programas de comercio exterior. El Consejo Superior de Comercio Exterior deberá sugerir al gobierno políticas de promoción de las mipymes ${ }^{10}$.

Otro grupo de acciones está enfocado a facilitar el acceso a los mercados. En ese sentido, la ley dispone que: "La Superintendencia de Industria y Comercio, con el fin de evitar que se erijan barreras de acceso a los mercados o a los canales de comercialización para las mipymes, investigará y sancionará a los responsables de tales prácticas restrictivas" ${ }^{11}$.

Finalmente, la ley complementa su promoción de las pymes por medio de tres acciones más:

La primera, se refiere al desarrollo tecnológico y al talento humano, en este sentido:

a) Se crea el Fomipymes, Fondo Colombiano de Modernización y Desarrollo Tecnológico de las micro, pequeñas y medianas empresas, cuyo objeto es la financiación de proyectos, programas y actividades para el desarrollo tecnológico de las mipymes y la aplicación de instrumentos no financieros dirigidos a su fomento y promoción. El Fomipymes tiene su aplicación en el agro por medio del Fondo de Inversiones de Capital de Riesgo de las Micro, Pequeñas y Medianas Empresas Rurales (Emprender).

b) Se articulan sistemas de información, como instrumentos de apoyo para la identificación de oportunidades de desarrollo tecnológico, de negocios y progreso integral.

9 CONGRESO DE COLOMBIA. Ley 590 de 2000 Art. 4 literales a, h, k. Diario Oficial No 44078, julio 12 de 2000. 10. CONGRESO DE COLOMBIA. Ley 590 de 2000, julio 10. Diario Oficial. No ${ }^{\circ}$.44.078, de 12 de julio 2000.

11 Ibíd. Art. 16. 
c) Se ordena al Sistema Nacional Ambiental (SNA) facilitar el acceso de las mipymes a la producción más limpia, la transferencia de tecnologías ambientalmente sanas, y el conocimiento y cumplimiento de las normas de protección y conservación del medio ambiente (Art. 27). Además, se pide al SNA adoptar las disposiciones conducentes a la flexibilización de los trámites para la obtención de las licencias ambientales en proyectos de las mipymes (Art. 28).

d) Los Centros de Desarrollo Productivo, al servicio de la microempresa, y los centros de investigación, al servicio de las mipymess, se incorporan al Sistema Nacional de Ciencia y Tecnología y a la Red de Centros de Desarrollo Tecnológico, coordinada por Colciencias.

e) Programas educativos y de creación de empresas para mipymes con participación del Icetex. En desarrollo de sus funciones, el Icetex destinará recursos y programas para facilitar la formación y el desarrollo del capital humano vinculado a las mipymes.

La segunda es el acceso a mercados financieros que se lleva a cabo de cinco maneras:

a) Préstamos e inversiones destinados a las mipymes. El gobierno nacional, en coordinación con el Banco de la República, podrá determinar temporalmente la cuantía o proporción mínima de los recursos del sistema financiero que, en forma de préstamos o inversiones, deberán destinar los establecimientos de crédito al sector de micro, pequeñas y medianas empresas.

b) Adquisición de títulos de emisión colectiva por parte de los fondos de pensiones. Estos podrán adquirir títulos de emisión colectiva por grupos organizados de mipymes que, a su vez, obtengan el respaldo de emisores debidamente inscritos y registrados, de conformidad con las disposiciones que regulan dichos fondos.

c) Condiciones especiales de crédito a empresas generadoras de empleo. El Fondo Nacional de Garantías podrá otorgar condiciones especiales a empresas generadoras de empleo por un $80 \%$ del valor del crédito requerido para el emprendimiento, de conformidad con el reglamento que expida el gobierno nacional.
La tercera acción se denomina creación de empresas, instrumentada a partir de:

a) Destinación de los recursos del artículo 51 de la ley 550 de 1999. Serán beneficiarios de los recursos destinados a la capitalización del Fondo Nacional de Garantías, en el artículo 51 de la ley 550 de 1999, todas las micro, pequeñas y medianas empresas, sin que para ello sea necesario que se acojan a lo establecido en dicha ley ${ }^{12}$.

b) Regímenes tributarios especiales. Los municipios, los distritos y departamentos podrán establecer regímenes especiales sobre los impuestos, tasas y contribuciones a las mipymes como exclusiones, períodos de exoneración y tarifas inferiores a las ordinarias.

c) Estímulos a la creación de empresas. Las pymes que surgieron después de la promulgación de la ley, tienen descuentos en las contribuciones parafiscales entre el 25 y el 75\%. Así, para el primer año el descuento era de $75 \%$, para el segundo de $50 \%$ y para el tercero del $25 \%$.

d) Líneas de crédito para creadores de empresa. Durante el primer trimestre de cada año, el Instituto de Fomento Industrial y el Fondo Nacional de Garantías establecen el monto y las condiciones especiales para las líneas de crédito y para las garantías dirigidas a los creadores de micro, pequeñas y medianas empresas.

Para completar la documentación de la política de apoyo, hay que mencionar el incremento de las líneas de crédito de fomento, ya que se autorizó a Findeter redescontar créditos a entidades de derecho privado y patrimonios autónomos. Además, se fortalecieron los mecanismos y modalidades de acceso a crédito de fomento al ser autorizada la banca de desarrollo (IFI, Bancóldex, Finagro, Findeter y FEN) para realizar el redescuento en contratos de leasing.

\section{BOGOTÁ Y LAS PYMES DE BASE TECNOLÓGICA}

En primera instancia es importante establecer el entorno económico-tecnológico de la ciudad para luego repasar el sistema de ciencia y tecnología de la capital. Por últi-

12 La ley 550 se conoce como la ley de quiebras pues se orienta a prestar asistencia a empresas que declarándose en quiebra tengan alguna oportunidad de salir de esa situación. En ese orden de ideas, la condición de que se está hablando, deja ver que las pymes, no obstante no se encuentren en las condiciones previstas en la ley 550, pueden acceder al apoyo del gobierno por medio de la ley 550 de 2000. 
mo se describen dos casos de análisis sectorial para la industria de artes gráficas y de desarrollo de software.

\section{Condiciones estructurales en Bogotá para las empresas de base tecnológica}

Según la Cámara de Comercio de Bogotá, la ciudad aporta la cuarta parte del PIB del país y genera tres millones de empleos que equivalen al $17 \%$ del total nacional. En Bogotá se asientan 180.000 empresas, de las cuales el $98 \%$ son pymes. El 10\% de las nuevas empresas que se asientan en la capital son manufactureras y el 34\% se dedican al comercio o son talleres de mecánica.

En términos de comercio exterior, por Bogotá ingresó el 55\% de las importaciones en el año 2002 y salieron el 28\% de las exportaciones nacionales. El principal destino son los Estados Unidos a donde se envía el 83\% de los productos exportados. El segundo mercado es la región andina.

A partir del sistema de indicadores de competitividad departamental ${ }^{13}$, se puede afirmar que Bogotá ocupa el primer lugar en el país. El mencionado estudio sobre competitividad ha distinguido siete factores que determinan en conjunto lo siguiente:

1. Condiciones de entrada. Se refieren principalmente a la dotación de factores, oferta ambiental y espacial, localización y niveles de acumulación de capital.

2. Condiciones de mercado. Centran la atención en los factores macroeconómicos tales como estructuras del mercado, nivel de exposición al mercado internacional y eficiencia y gestión empresarial.

3. Condiciones de transformación productiva. Entre otras se pueden señalar, la capacidad de generación y adopción de nuevas tecnologías, en especial las de conocimiento e información, y el manejo de sistemas de información.
4. Condiciones de sostenibilidad. Por ejemplo, en el uso de factores, como recursos naturales, o también sostenibilidad económica, lo que se refiere a la capacidad financiera, la organización social y política y el sistema institucional.

5. Condiciones de integración y cohesión social. Es decir, capacidad para construir proyectos compartidos de futuro a partir de la pertenencia a una ciudad, región o país.

6. Condiciones internas de negociación. Capacidad de crear espacios de participación y acuerdos sociales básicos entre los principales grupos de interés para el logro de metas compartidas de progreso y modernidad.

7. Condiciones de desarrollo. Entre otras, nivel y calidad de vida y condiciones de equidad en la distribución del ingreso.

Por otro lado, para llegar al concepto de competitividad regional se requieren dos conceptos que se complementan: competitividad estructural y competitividad revelada.

El primer subtipo de competitividad se refiere a la "potencialidad proporcionada por aquellos aspectos que le sirven como parámetros al crecimiento económico"14. Ocho fueron las variables que se tuvieron en cuenta para determinar el indicador de competitividad estructural. A modo de ejemplo se pueden citar: infraestructura productiva y localización, dotación de recursos naturales, empresas, innovación y tecnología.

El segundo subtipo de competitividad se denomina índice de competitividad revelada. Se considera un indicador ex post del desarrollo económico y del desarrollo de la región que permite observar los resultados alcanzados. Para este índice, la Universidad Nacional ${ }^{15}$ consideró tres categorías: crecimiento económico, competitividad externa y calidad de vida.

13 Phillipe de Lombard, José Guillermo García. “Sistema de Indicadores de Competitividad Departamental”. Universidad Nacional de Colombia-Centro de Investigaciones para el Desarrollo CID. Informe final. Proyecto equipo negociador "ALCA" en su componente de competitividad. Bogotá , 2002.

14 Ibíd. p. 69.

15 Ídem. 
Estos dos subtipos de competitividad fueron ponderados para generar el índice de competitividad departamental, que se define como: "el resultado logrado efectivamente en términos de crecimiento de la producción en el mercado, de ingreso real y de calidad de vida en un momento dado" 16 .

Dados estos factores y el Sistema de Indicadores de Competitividad Regional, desarrollado por la Universidad Nacional, se observa que:

1. El Índice de Competitividad Departamental (ICD) para Bogotá es 0,646, lo que le da el primer lugar. A continuación siguen, en su orden, Antioquia, Valle, Atlántico y Santander.

2. El Índice de Competitividad Estructural por departamentos (ICE) para Bogotá es 0,637. Le siguen, en su orden, Antioquia, Valle, Atlántico, Bolívar.

3. El Índice de Competitividad Revelada (ICR) para Bogotá es 0,670. Le siguen, en su orden, Valle, Antioquia, Santander y Atlántico.

Del extenso estudio que aquí se referencia, vale la pena resaltar, adicionalmente, las categorías correspondientes a empresas y a investigación y tecnología. En el primer caso, Bogotá como líder dobla a Antioquia, que es el segundo. En esta categoría se tienen en cuenta las variables: número de grandes empresas por cada mil habitantes, número de matrículas y renovaciones de empresas por habitante e inversión privada neta con referencia al PIB. Para el caso de innovación y tecnología, las diferencias son mucho menores aunque sigue Bogotá a la cabeza. Sin embargo, en variables como número de docentes doctores por cada mil habitantes o inversión pública en investigación, las diferencias son abismales.

Por todo esto, se puede considerar que Bogotá es el líder sin atenuantes en cuanto a competitividad en Colombia y se erige como centro natural de observación para este proyecto.

Sobre el desempeño económico de Bogotá en su dimensión industrial se pueden identificar por lo menos cinco particularidades (Obando, 2004): a. La estructura productiva es limitada para garantizar su sostenimiento competitivo a largo plazo. Este comportamiento se explica por una marcada orientación hacia el mercado interno y escasa penetración en el mercado internacional, además de las importaciones de bienes de capital, en tanto los empresarios, caracterizados por manejar sus empresas con fundamentos menos técnicos y más emotivos, históricamente han sido protegidos de la competencia, lo que los ha llevado a ser reacios a incorporar procesos innovadores y nuevas tecnologías.

b. La economía distrital se orienta hacia los bienes de consumo. Esta es una falla en la estructura productiva, ya que ante un episodio contractivo de la economía, el distrito se vería seriamente afectado, puesto que estos bienes tienen una alta elasticidad de ingreso y su oferta es netamente local (el 80\% de su demanda ocurre dentro de la ciudad).

c. La plataforma productiva local es limitada para incrementar sus niveles de competitividad en forma sostenible. Las razones que explican este hecho estriban en que la gran mayoría de las empresas $(78.15 \%)$ son pequeñas, se orientan al estrecho mercado interno y no generan recursos suficientes para innovar. Adicionalmente el problema empeora si se considera que la mayoría de las firmas son de carácter familiar por lo que es frecuente que no cuenten con los conocimientos técnicos, pero sí con un gran componente empírico en el manejo de procesos, tanto productivos como de gestión, donde la innovación surge más por casualidad que por procesos continuos de I + D.

d. Otra razón que ayuda a explicar esta situación es que las empresas tienden a crear ventajas en la reducción de costos por disminución de personal, en lugar de crear ventajas de largo plazo para aumentar la competitividad, incorporando tecnologías a los procesos y conocimiento en sus trabajadores.

e. Escaso apoyo del gobierno para orientar recursos y asesorías a los empresarios para la adquisición de bienes de capital de alta tecnología. Paralela a esta dificultad, la composición del PIB capitalino muestra que en los últimos años la producción de bienes de capital es decreciente, lo que indica que la industria bogotana no satisface la demanda por este tipo de bienes.

16 Ibíd. p. 71. 
f. El PIB distrital se ha especializado en servicios (60\%). Aquí se abren oportunidades de negocios en sistemas de información, redes, software especializado en gestión de procesos, etcétera.

En resumen, la economía distrital se centra en el mercado interno, produce principalmente bienes de consumo, su base productiva es una multitud de empresas pequeñas que no necesariamente (y explícitamente) están orientadas al I + D; agravando lo anterior no hay señales que indiquen que la tendencia cambie, en la medida en que la producción de bienes de capital está decreciendo. Sin embargo, en términos de servicios, el PIB distrital abre oportunidades de negocios en sistemas de información, redes, software especializado en gestión de procesos.

\section{El sistema de ciencia y tecnología distrital}

Las empresas de base tecnológica de Bogotá están rodeadas por un sistema de ciencia y tecnología distrital que inició su formación hacia el año 1992 (Obando, 2004).

En efecto, en la alcaldía de Jaime Castro se creó el plan estratégico Bogotá 2002 en el que se anuncian los lineamientos para convertir la ciudad en ejemplo de desarrollo, con mejor calidad de vida y líder en convivencia ciudadana.

Esta idea empezó a madurar en 1997 con un estudio sobre las condiciones de la economía distrital, que recalca la necesidad de incorporar el desarrollo tecnológico, la innovación y la competitividad, de manera que se oriente el plan de acción basado en una visión de largo plazo.

Hacia 1999 (Escobar, 1999 citado por Obando, 2004) se analizaron "las bases institucionales de un sistema distrital de ciencia y tecnología”. El análisis indagó la funcionalidad de la estructura administrativa del distrito respecto a las necesidades de desarrollo científico y tecnológico, así como los posibles esquemas institucionales apropiados para la gestión tecnológica de la cuidad.

Este estudio planteó un sistema distrital con cuatro objetivos: el diseño de instituciones de apoyo para la política de ciencia y tecnología (C y T), la propuesta de organización y articulación del sistema distrital de innovación, el sistema desde el ámbito gubernamental, y por último, ejemplos que confirman la construcción del sistema.

En ese mismo año, Amaya (1999) (citado por Obando, 2004) caracterizó la estructura tecnoeconómica de Bogotá y sus municipios periféricos y realizó el desarrollo del perfil tecnológico de las industrias más dinámicas entre los años 1990-1996. Allí se determinó que los productos químicos, plásticos, productos derivados del petróleo, industrias de metales no ferrosos, industria básica del hierro y del acero, fabricación de aparatos de transporte, construcción de aparatos eléctricos así como imprentas y productos alimenticios, son sectores hacia los cuales se orienta la industria del distrito.

También en 1999 (Jaramillo, 1999 citado por Obando, 2004) se estructuró la futura plataforma de conocimiento del distrito. Esta es el anillo de innovación que funciona como un polo atrayente y promotor de tecnología avanzada y altamente especializado, centrada en la zona de la avenida El Dorado que integra sistemas de producción avanzados. Su objetivo principal es la creación de un motor de desarrollo dentro del plan de ordenamiento territorial, por medio de la localización planificada de los centros de investigación, oferentes de conocimientos, empresas de bienes y servicios articulados.

Como complemento a la idea de innovación, la consultora Tecnos (1999 citado por Obando, 2004) diseñó una estrategia de $\mathrm{C}$ y T para Bogotá que establece el desarrollo de la base estructural para la elaboración de un plan de $\mathrm{C}$ y $\mathrm{T}$ para el distrito y proporcionó la base conceptual para reconocer los elementos relevantes de la política y caracterizar la demanda social por tecnología.

En 2000 se propuso un plan de acción e innovación para el distrito con el fin de modernizar tecnológicamente las empresas y crear condiciones que estimulen nuevos procesos de innovación sostenidos en el ámbito mesoestructural sobre cuatro aspectos: infraestructura institucional, oferta de recursos financieros, oferta de recursos humanos y generación y difusión de información. 
En 2002, Infante (citado por Obando, 2004) registró una modificación al sistema distrital de innovación, al crearse el centro distrital de producción e innovación "cuya función es el diseño y coordinación de la política distrital”. Asimismo, se planteó crear una oficina pública de orientación y desarrollo científico, tecnológico y para liderar la política tecnológica del distrito.

En el año 2000 se propusieron políticas que apoyaran la construcción de una base científica y tecnológica impulsora del proceso tecnológico. Para el año 2004 se contaba con una propuesta de agenda regional de $\mathrm{C}$ y $\mathrm{T}$ e innovación para Bogotá y Cundinamarca. Su objetivo era diseñar, mediante consenso de los diversos sectores interesados, el programa a corto, mediano y largo plazo para desarrollar las capacidades endógenas de ciencia y tecnología para la región.

Complementario a la anterior propuesta, se produjo el documento: "Ciencia, tecnología e innovación para la región" que analiza el proceso de desarrollo económico como un proceso sistémico y dependiendo de la innovación (ver Figura $N^{0} 1$ ).

\section{ALGUNOS ESTUDIOS DE PYMES DE BASE TECNOLÓGICA DE BOGOTÁ}

\section{La industria de artes gráficas}

Uno de los negocios en los que pueden darse pymes de base tecnológica se sitúan a lo largo de la cadena productiva del papel y de las artes gráficas. Dicha cadena cuenta con tres nodos (Malaver 2002a): materias primas (madera, bagazo, papel y cartón de reciclaje), pulpa de papel y cartón (papeles de imprenta y escritura, empaques, artículos sanitarios papeles especiales) y productos e impresos (por ejemplo, periódicos, cajas de cartón, pañuelos faciales, papeles para cigarrillos).
Esta cadena productiva se puede dividir en dos grandes negocios: la industria de la pulpa y el papel; y la industria gráfica. El primero se localiza en Cali, cerca de sus fuentes de materias primas. El segundo se concentra en Bogotá, cerca de sus mercados. En la industria gráfica predominan la intensidad de mano de obra y la presencia de pymes que, sin embargo, no captan la mayoría de la participación del mercado, pues ese lugar está reservado al grupo empresarial Carvajal (Malaver, 2002).

Según la revisión del estado del cluster, Malaver identifica en la dinámica empresarial de la industria, tres tendencias:

1. Creciente concentración de la propiedad en grupos económicos que han adquirido numerosas empresas independientes.

2. Adquisición de empresas impresoras por parte de grandes empresas trasnacionales.

3. Integración en el ámbito mundial de la industria gráfica, de la comunicación y el entretenimiento. Para el caso colombiano este fenómeno se ilustra en el grupo El Tiempo (CEET) y Carvajal.

Basado en un marco teórico fundado por ciertas ideas de Michael Porter ${ }^{17}$ y sobretodo por el enfoque de la gestión estratégica de la tecnología ${ }^{18}$, la investigación, centrada en la industria de las artes gráficas, indaga por tres puntos ${ }^{19}$ :

a. El nivel de actualización de las tecnologías utilizadas por las empresas del sector.

b. El grado de formalización de la gestión de estos recursos.

c. El conjunto de prácticas, que desde la perspectiva funcional asumida, definen el perfil de dichas capacidades.

$17 \mathrm{El}$ argumento desarrollado en torno a la competitividad en el libro Estrategia competitiva : técnicas para el análisis de los sectores industriales y de la competencia de Michael E. Porter. México: Editorial Continental.

18 Según Tidd (2000 citado por Malaver, 2002): "la competitividad no proviene de los productos sino de las capacidades distintivas de la empresa. Dado que estas solo son potencialidades, la función de la gestión tecnológica es materializarla a través de la innovación." p. 58.

19 İ́bid.. p. 60. 


\section{FIGURA № 1. Línea de tiempo del proceso}

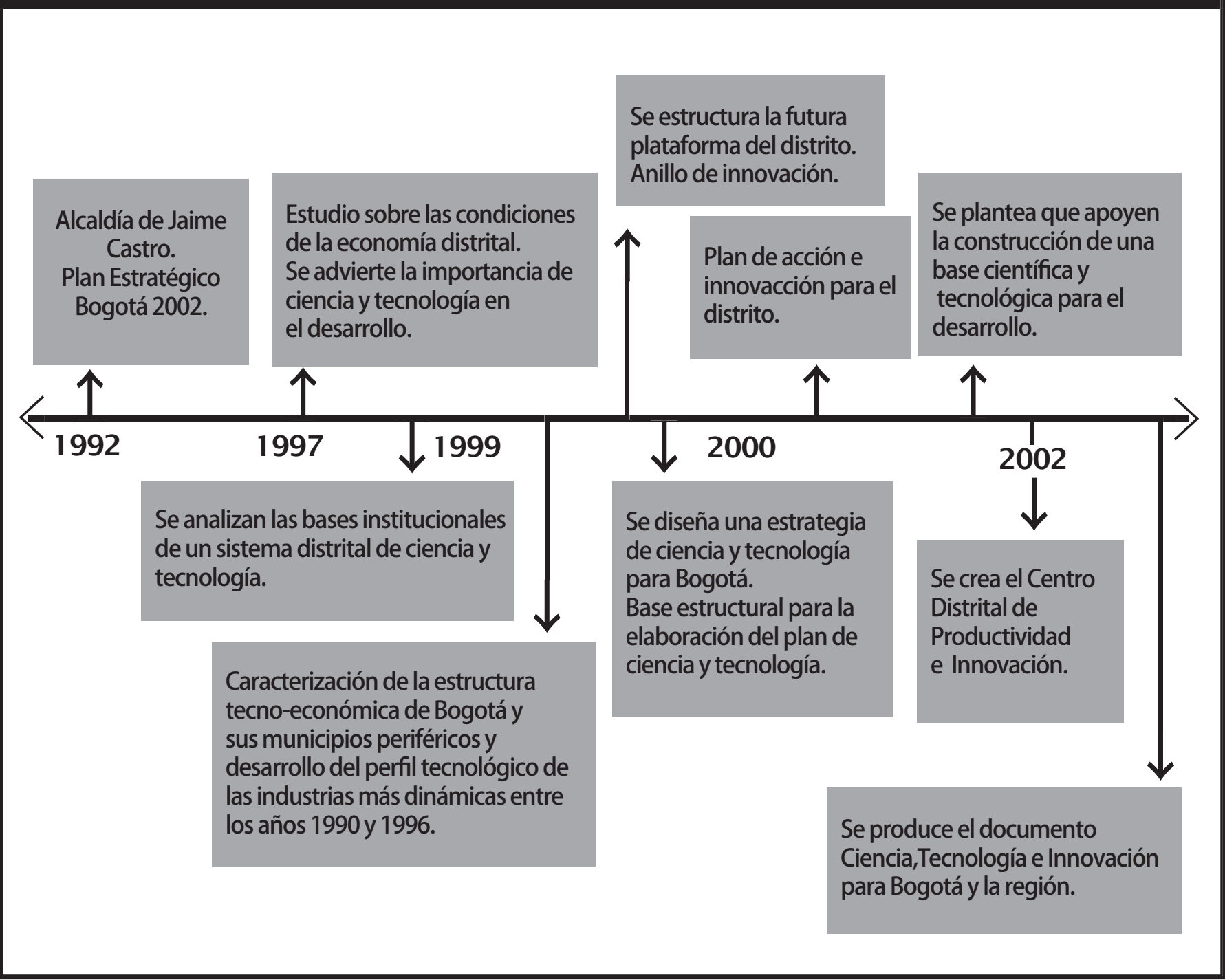

Los resultados que se encontraron fueron los siguientes:

Con respecto a la actualización tecnológica, la industria gráfica tiene tres características estructurales (Malaver, 2002):

a. Es usuaria de los desarrollos tecnológicos de otros países, por lo que su dinámica tecnológica se somete al ritmo de incorporación de los mismos. b. La modernización tecnológica es bastante diferenciada incluso en el interior de las mismas empresas.

c. En medio de esta heterogeneidad existe un marcado atraso tecnológico ya que la tecnología que se compra es usada y solo una pequeña parte es de última generación.

Con respecto a la formalización de la gestión de recursos, Malaver encontró que: 
No hay dentro de la estructura organizacional de las empresas estudiadas, espacio específico asignado para la gestión de la tecnología. Son casi inexistentes los departamentos de I+D o la asignación de recursos (humanos y financieros) específicos para el desarrollo de las capacidades tecnológicas de las empresas; no existen estrategias tecnológicas explícitas, ni conciencia de la conexión entre estas y las estrategias competitivas.

Para la mayoría de las empresas el uso de la tecnología es "funcional" "táctico" de mera adecuación a las estrategias competitivas. Sin embargo, dado que la gerencia tiene conocimientos de producción y mercadeo, aquellas se interesan por estar al tanto de los avances tecnológicos de su sector.

Finalmente, en lo que se refiere a las capacidades tecnológicas, las empresas presentas tres defectos:

a. Atraso tecnológico. Su origen se debe a que se adquiere tecnología usada, con poca proporción de tecnologías digitalizadas, bajo usufructo de las tecnologías de la comunicación e información, así como alta heterogeneidad tecnológica incluso en empresas avanzadas.

b. Manejo informal. Dada esta condición en las empresas, el cambio técnico se mira desde la barrera en una actitud de expectativa mientras se verifican los resultados de la aplicación de la misma.

c. Poco valor estratégico dado a los recursos. Se refiere a que la mejora tecnológica (como se mencionó arriba) sirve a manera de afianzamiento de la posición en un mercado existente, pero no en una concepción de planeación estratégica que dé pie para considerar la innovación como una herramienta para la generación de nuevos mercados basados en ventajas competitivas.

\section{La industria de desarrollo de software}

El negocio del desarrollo de software se puede entender como una cadena productiva. En tal sentido, la Cámara de Comercio de Bogotá (CCB) publicó recientemente un estudio sobre el balance tecnológico de esta cadena productiva (CCB, 2006 en línea).

Se entiende por cadena productiva de desarrollo de software aquella compuesta por una base de cuatro elementos generales y factores del entorno:
Insumos: programas de software (código fuente), hardware, infraestructura de telecomunicaciones, fundamentos de computación y recurso humano.

Gerencia de software: se refiere a la administración y gestión de proyectos e incluye: calidad, adquisición de software, configuración y procesos de riesgos.

Ingeniería de software: ingeniería de requerimientos, diseño del software, codificación, ensayo, operación y mantenimiento.

Comercialización: algunas de las formas como se hace son: paquetes de software, reingeniería, outsourcing, bodyshopping, offshore y (principalmente en Colombia) la construcción a la medida.

En la base de la cadena está el business intelligence, el cual permite reconocer, además, nuevos negocios en robótica, nanotecnología, inteligencia artificial y bioinformática.

Como factores del entorno están: el Gobierno, la Cámara de Comercio, las asociaciones, las instituciones para el fomento de la industria del software. En tanto elementos de apoyo: universidades, instituciones técnicas y tecnológicas y dominio del idioma inglés. Como reguladoras de las prácticas de producción las normas CMM y la ISO.

\section{Características de la industria del software en Colombia}

A modo de resumen, se intentará recoger el desarrollo del tema presentado en el estudio de la Cámara de Comercio de Bogotá (ver Cuadro $\mathrm{N}^{\mathrm{O}} 1$ ).

El trabajo realizado por la Cámara de Comercio de Bogotá trataba, en últimas, de identificar brechas tecnológicas de la industria nacional y, en especial, de Bogotá con respecto a la competencia internacional. A partir de la metodología de análisis de la firma consultora Qubit Cluster: metodología Compstrat ${ }^{\mathrm{TM}}$, para identificar la estructura de la cadena productiva alrededor de los llamados conglomerados (clusters); MapTec ${ }^{\mathrm{TM}}$ para la identificación de las brechas tecnológicas entre los procesos medulares y las tecnologías usadas por las empresas locales respecto al estado del arte de la ca- 


\section{CUADRO № 1. Características de la industria del software}

\section{en Colombia}

\begin{tabular}{|c|c|}
\hline No de empresas a $2001^{*}$ & 4.000 \\
\hline Ocupación ${ }^{* *}$ & $\begin{array}{l}\text { a. Desarrollo de software } \\
\text { b. Comercialización de hardware } \\
\text { c. Consultoría }\end{array}$ \\
\hline Volumen de negocios & 0.15\% de las exportaciones no tradicionales (US\$13.000 millones) en 2001 \\
\hline Innovación & $\begin{array}{l}\text { El grado de innovación propio es bajo; en tanto es preferible comprar un produc- } \\
\text { to creado y probado en el exterior que arriesgarse a la creación nacional. }\end{array}$ \\
\hline Especialidad & $\begin{array}{l}\text { Desarrollos a la medida. No hay asociatividad, dado la confidencialidad y el deber } \\
\text { de cumplir con derechos de autor. Eventualmente hay asociaciones de empresas } \\
\text { que complementan servicios. Trabajan unidas durante el proyecto y el resto del } \\
\text { tiempo por separado. }\end{array}$ \\
\hline Recurso humano & $\begin{array}{l}\text { Es la pieza más influyente. Lo constituyen principalmente: ingenieros de siste- } \\
\text { mas, tecnólogos en diferentes áreas y técnicos en diferentes especialidades. }\end{array}$ \\
\hline Principales clientes & $\begin{array}{l}\text { Clientes finales: compran productos más que servicios. Son anónimos. Compran } \\
\text { licencias de software, suministros, equipos de computo, etc. } \\
\text { Empresas usuarias de tecnología: compran por licitación o programas de com- } \\
\text { pras, dado un estudio de necesidades. Son conocedores, exigentes y se asesoran } \\
\text { de expertos para la contratación. Les interesa el servicio, mantienen comuni- } \\
\text { cación directa con el proveedor y lo presionan para hacerlo cumplir estándares } \\
\text { internacionales. } \\
\text { Entidades estatales: se guían por legislación propia e incluyen en sus contratos } \\
\text { estándares internacionales. }\end{array}$ \\
\hline $\begin{array}{l}\text { Principales competidores } \\
\text { internacionales }\end{array}$ & $\begin{array}{l}\text { América Latina } \\
\text { Chile apoyado por el gobierno con el Fondo de Fomento del Desarrollo Científi- } \\
\text { co y Tecnológico - FONDET, se ha enfocado en infocomunicación (v. gr. informá- } \\
\text { tica educativa, aplicaciones en banda ancha para mejor uso de redes, creación } \\
\text { de red universitaria nacional, desarrollo de software para diversas aplicaciones } \\
\text { industriales). } \\
\text { Costa Rica, también orientada por políticas públicas, cuenta con programas de } \\
\text { investigación y desarrollo en el área tecnológica. } \\
\text { Resto del mundo } \\
\text { India: su característica principal es el bodyshopping, es decir, llevar al experto a } \\
\text { la tierra de residencia del cliente. Esta actividad representa el } 60 \% \text { de sus ventas. } \\
\text { Irlanda: se dedica a la adaptación y localización de software empaquetado. Ade- } \\
\text { más trabaja el área de servicios especialmente internet y consulta multimedia. } \\
\text { Estados Unidos: industria de servicios no de producto. Brindan soluciones } \\
\text { completas, (por ejemplo, e-commerce) integración de aplicaciones en internet, } \\
\text { conversión a modelos basados en internet y desarrollo y mantenimiento de sitios } \\
\text { web. }\end{array}$ \\
\hline \multicolumn{2}{|c|}{$\begin{array}{l}\text { * Según Fedesoft (2001). } \\
\text { ** Las empresas del sector se dedican a dos o más actividades. }\end{array}$} \\
\hline \multicolumn{2}{|c|}{$\begin{array}{l}\text { Fuente: Elaboración propia del autor basado en: CÁMARA DE COMERCIO DE BOGOTÁ (2005). Balance tecnológico } \\
\text { cadena productiva desarrollo software en Bogotá y Cundinamarca. }\end{array}$} \\
\hline
\end{tabular}


dena productiva, según las mejores prácticas empresariales mundiales; y PTT $^{\mathrm{TM}}$, metodología para definir el plan tecnológico estratégico a seguir en el cierre de las brechas identificadas, el documento establece las siguientes problemáticas:

- Los clientes locales están demandando desarrollos a la medida.

- Pocas empresas de consultoría disponen de habilidades en reingeniería.

- Faltan estrategias de comercialización especialmente orientadas al mercado externo.

- Ausencia de metodologías para la definición de necesidades de los clientes.

- Falta actualización tecnológica en técnicas de diseño.

- La gestión empresarial no considera relevante definir las métricas.

- Ausencia de programas de codificación y prueba y de sus métricas.

- Actualización de los equipos (hardware).

- Falta la infraestructura adecuada en telecomunicaciones.

- Falta implantación de normas de calidad.

- Manejo de conceptos generales de administración.

- Especialización del recurso humano.

- Certificaciones.

- Manejo del idioma inglés.

\section{DESARROLLOS TEÓRICOS Y EJERCICIOS EMPÍRICOS SOBRE LA INTERNACIONALI- ZACIÓN DE EMPRESAS EN BOGOTÁ}

En el caso colombiano hay al menos dos grandes perspectivas para el estudio del tema de la internacionalización: una es la que se centra en reinterpretar teorías extranjeras para estudiar los procesos de internacionalización, y otra, basarse en teorías para mejorar el desempeño de las empresas en cuanto a internacionalización se refiere. En otras palabras, un grupo de teóricos se centran en estudiar el hecho objetivo de la internacionalización en Colombia, y el otro grupo hace elaboraciones teóricas para que los empresarios cualifiquen sus procesos de internacionalización. Con respecto al primer grupo de desarrollos, hay teorías para explicar los hechos. Específicamente se pueden mencionar trabajos desde la perspectiva evolucionista y modelos de planeación sistemática con diferentes matices.
En relación con los modelos de planeación sistemática, el primero a revisar es una aplicación de un modelo norteamericano de predicción del potencial exportador para el caso de las pymes colombianas. El modelo guía (original) es un modelo probabilístico para identificar el potencial exportador de empresas no exportadoras. Uno de los supuestos claves de este modelo es que las empresas llegan a un determinado punto en el tiempo llamado momentum en que las empresas creen que tienen las condiciones para la expansión territorial; es decir, creen que han acumulado las suficientes habilidades para expandir mercados. Así, el modelo original llega a plantear que el comportamiento exportador es el resultado de la intensión exportadora, la cual, a su vez, está en función de la habilidad para expandir mercados, ya que el interés está en medir la intensión que tiene una empresa no exportadora en comenzar un proceso exportador, se plantean dos preguntas:

¿La empresa cuenta con un plan de exportación para los próximos dos años? Y ¿la empresa planea invertir sus recursos en mejorar su nivel de ventas internacionales? Detrás de las anteriores preguntas subyacen dos hipótesis de partida: la primera es: "Entre los no exportadores, la habilidad para expandir los mercados de la empresa está positivamente relacionada con su intensión exportadora”.

La segunda hipótesis se refiere a la actitud de las empresas dada la presencia de barreras externas (tarifarias, no tarifarias, de tasa de cambio), operacionales (dificultad para recibir pagos, coordinación en el transporte, etcétera) e internas (falta de compromiso gerencial, recursos humanos y financieros insuficientes, etcétera). La hipótesis planteada fue: "Entre los no exportadores, a mayor intensión exportadora, más importancia se le da al querer superar las barreras" (Laurerio, Marín, 2004, p. 11).

Para la aplicación al caso colombiano, las autoras citadas mantuvieron en general la estructura del modelo guía, pero añadieron un tercer factor explicativo denominado "perfil del gerente", toda vez que "el nivel de educación, la experiencia internacional previa y las actitudes específicas hacia la internacionalización pueden definir el comportamiento exportador de una empresa" (Burton, Schiegelmich, 1987 citados por Laurerio, Marín, 2004 p. 15). 
Los resultados se obtuvieron a partir del procesamiento de la información de 104 respuestas de empresas ubicadas principalmente en el sector manufacturero (85\%) y en sector de servicios. En general, se llegó a la conclusión de que el tamaño de la firma no es una limitación en el desempeño exportador, pues el tamaño no limita la propensión a exportar. De otro lado, la investigación afirma haber demostrado que las variables de la habilidad para expandir mercados explican, en mayor medida, el potencial exportador de una empresa ya que se encontró una relación positiva y en muchos casos significativa entre los indicadores de desempeño exportador y el comportamiento pasado en la expansión de las firmas, así como entre el desempeño exportador y las capacidades competitivas (Laurerio, Marín, 2004 p. 54).

Otra aplicación de la teoría de la planeación sistemática es la construcción del perfil de la pyme exportadora exitosa (Moori 2004). Para lograr generar tal perfil, este trabajo se dividió en dos partes: en la primera construyó un índice llamado Indicador del Desempeño Exportador (IDE) a partir de un grupo de empresas de industria manufacturera de exportaciones no tradicionales. Las empresas se clasificaron en tres grandes grupos según su tamaño (micro, pyme y grande). El índice se alimentó de cuatro variables: continuidad en el negocio exportador (exportar sistemáticamente), dinamismo exportador (evolución creciente de las ventas externas), diversificación en mercados externos (número de mercados en los cuales hay presencia) y complejidad de los mercados externos (mercados de difícil acceso).

Los resultados por componentes del IDE fueron:

- Poco más de la mitad exportó en solo uno o dos años del periodo analizado (1995-2003).

- Cerca de dos tercios registró una caída o estancamiento en las ventas externas.

- La mayoría exportó a menos de dos mercados (55\% a un mercado y $18 \%$ dos).

- Cerca de dos tercios dirigió la mayor parte de sus ventas a mercados considerados de difícil acceso.

En cuanto a los resultados generales de la aplicación del índice IDE se tienen los siguientes resultados:
- Existe un grupo minoritario de pymes que ha obtenido resultados exitosos en mercados externos (19\% del total de agentes pyme).

- Su contribución al monto exportado del estrato es muy significativa. Las pyme exportaron el 57\% del total.

- El monto promedio exportado por firma es superior. Seis veces mayor. Pasó de 0,07 a 0,41 millones de dólares.

En la segunda parte evaluó las prácticas microeconómicas de las pymes a partir de indagar sobre elementos que hacen la diferencia al momento de exportar. Los aspectos indagados fueron:

1. Atributos generales de las empresas.

2. Dimensiones que repercuten en el negocio exportador: estructura, canales de venta y promoción comercial.

3. Grado de utilización y conocimiento de instrumentos de fomento.

4. Desarrollo de competencias endógenas.

Algunos de los resultados más destacados con respecto a los aspectos indagados fueron:

\section{Atributos generales.}

- La pyme exitosa tiende a ser más joven (15 frente a 21 años).

- La pyme exitosa tiende a exportar desde el principio.

- El 60\% de las pymes exitosas incrementaron sus ventas totales frente a $25 \%$ del resto.

\section{Dimensiones que repercuten en el negocio exportador: estructura, canales de venta y promoción comercial.}

- Contrasta el altísimo peso de Proexport y los viajes exploratorios como fuentes de oportunidades comerciales, con respecto al resto de opciones como la internet, la solicitud del cliente, las cámaras de comercio. Estas últimas son prácticamente el 10\% de las oportunidades para las pymes.

3. Grado de utilización y conocimiento de instrumentos de fomento.

- Ambos aspectos no diferencian a las pymes exitosas del resto.

- La mayoría de los exportadores que utilizó instrumentos asignó un nivel alto de utilidad. 


\section{Desarrollo de competencias endógenas.}

- El 75\% de las pymes exitosas tiene un nivel entre elevado e intermedio respecto de competencias tecnológicas frente al $43 \%$.

- El 78\% de las pymes exitosas tiene un nivel entre elevado e intermedio respecto de competencias comerciales frente al 37\%.

Un trabajo teórico práctico más ecléctico en cuanto a los enfoques teóricos, pero que basa sus ejercicios empíricos en el estudio de casos y no en análisis estadístico de muestras, es el realizado por Trujillo et al. (2006). Este trabajo consiste en realizar una revisión de las teorías de internacionalización que no necesariamente se circunscriben a la pyme. El análisis de las teorías llevó a los autores a agruparlas en tres grandes grupos así: perspectiva económica, de proceso y redes.

La perspectiva económica se caracteriza por entender que la internacionalización hace parte de la estrategia empresarial; es el resultado de la toma de decisiones racionales que busca la mejor relación beneficio-costo, por lo cual, el directivo actúa como analista y normalmente escoge la inversión extranjera directa como modo de entrada.

Desde la perspectiva del proceso, la internacionalización es fruto de razones económicas y actitudinales donde se toman decisiones racionales e intuitivas en cada una de las etapas del proceso. Desde este enfoque, otra variable explicativa es la similitud con los mercados locales "menor distancia psicológica" y se pueden tomar todos los modos de entrada, pero en forma progresiva. Aquí el directivo es más bien la persona que posee la experiencia y el conocimiento.

Finalmente, en el enfoque de redes, la internacionalización es fruto de la ventaja competitiva que ofrecen las redes por las sinergias empresariales. El directivo posee los contactos, por lo cual, la cooperación es el modo de entrada habitual.

En cuanto al aspecto práctico, este documento de investigación se centra en el sector de los servicios públicos domiciliarios en Colombia. En este sentido, se revisa la experiencia de ISA (Interconexión Eléctrica
S.A) EPM (Empresas Públicas de Medellín) la Triple A de Barranquilla y el caso central que es la EAAB (Empresa de Acueducto y Alcantarillado de Bogotá). Como conclusión interesante de este análisis de casos Trujillo et al. plantean que los tres casos reflejan los puntos en los que las tres teorías convergen. En consecuencia, los tres casos muestran que son cuatro los elementos que caracterizan su internacionalización: el primero, es una ventaja competitiva exclusiva basada en la cadena de valor; el segundo, la favorabilidad del entorno macroeconómico; el tercero, la existencia de incentivos económicos; y el cuarto, que la internacionalización actúa como parte de la estrategia empresarial.

Un caso distinto es el que ocurre en la EAAB donde se realizó un análisis que intentaba contestar a la pregunta sobre si esta empresa debería o no empezar un proceso de internacionalización.

Para responder a la pregunta, el trabajo analizó las variables de la internacionalización soportados en los grupos de teorías revisadas aunque sobresale, como eje central, un cuarto soporte teórico: la ventaja competitiva de Michael Porter. De esta manera se hace un estudio de las condiciones del entorno regional y global y luego un balance de las fortalezas y debilidades internas a partir de un análisis de cadena de valor.

La respuesta final a la pregunta es afirmativa siguiendo los lineamientos propuestos por el enfoque económico: "...puesto que cuenta con las ventajas competitivas sostenibles, el proceso de internacionalización hace parte de la planeación estratégica y la relación beneficio costo será evaluada por la empresa Aguas de Bogotá para cada oportunidad de negocio" (Trujillo et al. 2006, p. 20).

Un análisis de casos enfocado a pymes de base tecnológica es el realizado por Castro, Chavarro y Ramírez (2006). En él se hace una revisión teórica exhaustiva y se intenta revisar las características generales de las prácticas de internacionalización de algunas empresas en Bogotá a partir de la construcción de un índice de internacionalización. Dado que los autores de este artículo son los mismos que hicieron la investigación que se reseña, nos permitimos hacer una cita extensa de las conclusiones generales obtenidas. 
Así, a partir de la revisión, se encontró que la propuesta holista recogía lo esencial de los planteamientos más recientes y que era frecuentemente reseñado en las revisiones empíricas sobre internacionalización de pymes de base tecnológica. Además es de destacar que las aproximaciones de la interfase mercadeo emprendimiento y la perspectiva de redes se perfilan como las hipótesis de trabajo de mayor proyección empírica (Fillis, 2001). En este orden de ideas, el cuestionario fue diseñado con el fin de recoger la mayor cantidad de información aportada por el modelo holista, dado su carácter transversal en los modelos recientes.

Sin embargo, a la luz de los datos y las entrevistas con los empresarios, observa esta investigación que las explicaciones teóricas más ajustadas con la realidad de las empresas estudiadas, son: (admitiendo que es una extraña combinación) la interfase mercadeo emprendimiento y en menor medida el modelo Uppsala. En efecto, se aprecia que una marcada aversión al riesgo por parte de muchos empresarios (quienes aún en muchos casos tienen en mente que primero se debe tener una sólida posición en el mercado interno para luego intentar salir) inhibe las iniciativas de incursión internacional a cualquier nivel de la cadena de valor, más que una deficiente organización interna de la empresa.

Por otro lado, cuando se les pidió a los empresarios que contaran su experiencia particular de internacionalización, la gran mayoría coincidió en que el origen de su inserción internacional fue muy dependiente de la casualidad o de oportunidades no fabricadas conscientemente. Hay casos en los que, dados contactos previos de carácter académico (relación profesor-estudiante), se le plantea a la empresa que intente un envío o haga de determinado proyecto, pero bajo la premisa de probar suerte. A pesar de las múltiples tentativas, tan sólo unas pocas se traducen en casos exitosos que llegan a ser un flujo relativamente continuo de venta. En otros casos, familiares de los productores van a otro país y se llevan muestras para comercializar en pequeñísimas escalas. Dependiendo de la aceptación, la empresa se anima a formalizar el flujo de producción hacia el exterior.

En el estudio también se buscó un contraste entre las empresas que hicieron parte de la selección a través de un instrumento de indización que, si bien no corresponde a una modelación formal, por su base cualitativa, sí permite dar información que traducida en números, compara el desempeño relativo de cada una de ellas y lo refleja en un ranking que abarca no solamente el rompimiento de barreras transfonterizas y culturales, sino que también permite ver si la estructura operativa y administrativa ha incidido en este proceso.
Se ha construido esta herramienta de manera flexible, de tal manera que se pueda posteriormente complementar el estudio, con otras variables que se consideren relevantes. A través de las categorías establecidas es posible catalogar las nuevas variables, que serían ponderadas de acuerdo con el orden de importancia planteado y explicado en el documento.

La hipótesis que se quiso contrastar con el instrumento, era que el éxito o fracaso en la internacionalización de una pyme de base tecnológica estaba directamente explicado (o habría una correlación fuerte) por el manejo administrativo y operativo de la misma. Sin embargo, este recurso no nos permitió corroborar la afirmación puesto que empresas con relativo éxito en la inserción internacional no necesariamente obedecían a buenos esquemas de administración y también se dio el caso de empresas, que a pesar de contar con andamiajes operativos y administrativos buenos, no logran un impacto internacional significativo. La explicación alternativa surge del perfil del empresario en la medida en que este esté dispuesto a correr riesgos y también por efectos de coyuntura que se revierten en negocios transfronterizos. Por esta razón encuentra que el modelo de interfaz emprendimiento-mercadeo como el más adecuado para explicar el comportamiento de las empresas de base tecnológica. (Castro et al. 2006, p. 75 a 77).

Una vez revisados los distintos matices de los desarrollos de la planeación sistemática como teoría de internacionalización en Colombia, y los enfoques ya no tan apegados a un solo esquema teórico para explicar la internacionalización como los de Castro, Chavarro y Ramírez (2006), queda por revisar otra orilla teórica discutida en el país como es la visión holística que mezcla una teoría económica como la economía evolutiva y una empresarial como el paradigma basado en recursos. Esta visión comprensiva de ambas teorías se llama Dirección por Competencias Básicas Distintivas (DCBD), de acuerdo con Uribe (2001). Con esta propuesta se pretende comprender cómo las empresas seleccionan el modo más apropiado de entrada a los mercados extranjeros o lo que es lo mismo, cómo fijan sus límites organizacionales al otro lado de las fronteras nacionales a partir de los recursos y las capacidades que poseen (Uribe, 2001, p. 25).

Desde DCBD se afirma que la internacionalización es un proceso lento y gradual de compromiso con las actividades de venta y producción foráneas. El avan- 
ce en el proceso está asociado con el ajuste entre los recursos y las capacidades disponibles en la empresa y los estándares que establece el mercado extranjero, y además, la necesidad de adquirir y acumular conocimiento en forma creciente y dinámica. Dado que se supone que la sección del modo de entrada es el resultado del cálculo de las condiciones relacionadas con el desarrollo y el despliegue de recursos y capacidades de la empresa, se tiene que el análisis sigue criterios como (Uribe, 2001):

- Para que la empresa pueda competir con éxito en el mercado actual es indispensable que tenga más de una capacidad o mejor un conjunto de capacidades complejas.

- La fuente de las capacidades puede estar enraizada en la empresa, el sector o el país. Si la empresa opera en ambientes diversos puede obtener una base de conocimientos superior que la capacita para competir con mayores posibilidades de éxito.

- El acercamiento y el despliegue de recuros-capacidades específicas a un mercado extranjero conlleva un proceso lento y costoso que es necesario minimizar sobre todo en relación con los competidores experimentados que están ya presentes en ese mercado.

- La experiencia que acumula la empresa a través de las operaciones que realiza en mercados extranjeros es valiosa. Cuando la transferencia, aplicación, adaptación y acumulación de los recursos- capacidades tiene resultados exitosos, la empresa tenderá a replicar la experiencia.

En contraste con este conjunto de perspectivas que intentan explicar comportamientos, a partir de postulados teóricos, hay un reducto de publicaciones que tratan el problema de la internacionalización, no tanto a partir del análisis de hechos objetivos, sino a partir del deber ser; es decir, hay algunas propuestas orientadas a mejorar el desempeño de las decisiones de internacionalización de los gerentes.
Como ejemplos de esta corriente hay trabajos monográficos y algunos artículos en revistas universitarias como las siguientes:

- La Universidad Jorge Tadeo Lozano a través del grupo Asesor G-15 escribe en el año 2000 La internacionalización de la pyme colombiana ${ }^{20}$. Este trabajo es un libro guía que describe herramientas y conceptos clave para la internacionalización de empresas como planes de mercadeo y estrategias de entrada a mercados extranjeros.

- Un ejemplo de lo que se publica en revistas universitarias es el trabajo de Uribe (1998) el cual elabora una base para el diseño de las estrategias de internacionalización empresarial a partir de las formas de entrada a los mercados extranjeros: "Las formas de entrada a los mercados extranjeros: base para el diseño de las estrategias de internacionalización empresarial" ${ }^{21}$.

A modo de resumen de este apartado, se puede decir que los desarrollos teórico-empíricos de la internacionalización de pymes en Bogotá parece tener dos grandes corrientes: la primera centrada en explicar y documentar alguna(s) teoría(s) particulares de internacionalización; enfocada en los hechos objetivos ; y otra corriente, en hacer que los responsables de internacionalizar empresas tomen decisiones con criterios más amplios y certeros. En otras palabras, centrados en el deber ser de la internacionalización. Es posible que puedan existir otros enfoques o preocupaciones en la bibliografía nacional sobre internacionalización, pero la revisión hecha por los autores (con las limitaciones que siempre existen en estos casos, como la imposibilidad de abarcar todas las universidades y fuentes bibliográficas, o aún teniendo acceso a todas ellas, la imposibilidad de leer y comprender toda la información disponible), da para pensar que esta es una presentación general que no agota el tema, pero sí lo cubre en una parte significativa.

20 GRUPO ASESOR G 15, (2000). “Internacionalización de la Pequeña y Mediana Empresa Colombiana” Monografía 264, Facultad de Comercio Internacional, Bogotá: Universidad Jorge Tadeo Lozano.

21 Cuadernos de Administración. Bogotá: Pontificia Universidad Javeriana. № 21. 


\section{CONSIDERACIONES FINALES}

La bibliografía revisada sobre internacionalización orientada a discutir teorías y documentar realidades a partir de ejercicios empíricos, tiene tres matices: uno son los trabajos principalmente empíricos (Laurerio Marín, 2004) y (Moori, 2004), otros que relacionan la teoría con la realidad colombiana a partir de casos (Trujillo, 2006) y (Castro, Chavarro y Ramírez, 2006) y finalmente, trabajos que son principalmente discusiones teóricas (Uribe, 2001).

Existe un relativo desinterés por estudiar las pymes de base tecnológica, por los menos en Bogotá. Es lamentable ver que se hace un énfasis en comprender procesos y dinámicas en sectores industriales ya estudiados, y marginalmente, muy poco énfasis en las pymes de base tecnológica (este comentario incluye también lo referente a internacionalización, aunque no sea difícil mostrar que es un grupo de empresas con experiencias administrativas llamativas, enriquecedoras y dinámicas de negocio comercialmente prometedoras a la luz de procesos de globalización.

Gran parte de la bibliografía revisada todavía tiene una visión parcial de lo que significa internacionalización. En general, se observa que este término se reduce a exportación e importación de bienes, y si acaso, a servicios dejando de lado procesos adicionales como inversión extranjera directa, licencias, empresas de riesgo compartido, internacionalización de partes de la cadena de valor distintas a la comercial como por ejemplo, la de investigación, desarrollo e innovación (I\&D+I) o la de financiación, por solo mencionar algunos ejemplos.

\section{BIBLIOGRAFÍA}

-ASOCIACIÓN PARA EL DESARROLLO REGIONAL DE CANTABRIA. Programa regional de acciones innovadoras. Bases del concurso 2004. En línea: http:// www.sodercan.com/documentos/Otros/BasesConcursoEBT2004.doc 20/12/04

-CÁMARA DE COMERCIO DE BOGOTÁ 2005. Balance Tecnológico Cadena Productiva Desarrollo Software en Bogotá y Cundinamarca. En línea: http://competitividad.ccb.org.co/documentos/2006_2_20_14_17_35_ BT\%20Software.pdf 04/07/06

-Casa Editorial El Tiempo Bogotá (Colombia), Empresa de Teléfonos de Bogotá. "Pymes: La empresa día a día. Manejo de situaciones cotidianas y su entorno". Bogotá: Portafolio.

-CONGRESO DE LA REPÚBLICA. Ley 590 de 2000. Diario Oficial $\mathrm{N}^{\mathrm{o}}$. 44.078, 12 de julio, 2000. Modificada por la ley 905 de 2003.

-EAN - ACOPI - CINSET - DANE, Observatorio Económico para la Pyme Colombiana (2004). Las pequeñas y las medianas empresas en Colombia 1992 - 2001: De la crisis al resurgimiento. Bogotá.

-FILLIS, Ian (2001). "Small firm internacionalisation: An investigative survey and future research directions". Management Decision. London. Tomo 39, No 9.

-GRUPO ASESOR G 15, (2000). "Internacionalización de la Pequeña y Mediana Empresa Colombiana” Monografía 264, Facultad de Comercio Internacional, Bogotá: Universidad Jorge Tadeo Lozano.

-LOMBARD, Phillipe de. y GARCÍA, José Guillermo (2002). "Sistema de Indicadores de Competitividad Departamental". Informe final proyecto equipo negociador "ALCA" en su componente de competitividad. Bogotá: Universidad Nacional de Colombia-Centro de Investigaciones para el Desarrollo-CID.

-MALAVER, F. (2002a). "Un perfil de las capacidades tecnológicas en la industria de las artes gráficas, imprentas y editoriales". Innovar. Bogotá: Universidad Nacional de Colombia. $\mathrm{N}^{\circ} 20$, julio-diciembre.

-MALAVER, F. y ZERDA, A. (2003). La innovación en la industria colombiana. Bogotá: Centro Editorial Javeriano (Ceja)-OCYT-Colciencias.

-MARTÍNEZ, Daniella y MARÍN, Alejandro (2004). "Predicción del potencial exportador de las pymes colombianas". Serie mejores trabajos de grado $N^{\circ} 81$. Bogotá: Universidad de los Andes.

-MOORI KOENIG, Virginia (2004). Perfil de la pyme exportadora exitosa. El caso colombiano. Bogotá: Fundes Colombia. 
-OBSERVATORIO DE CIENCIA Y TECNOLOGÍA. Glosario http://www.ocyt.org.co/ind/pfd/B15.pdf 20/12/04 -OBANDO RODRÍGUEZ y VELASCO ÁLVAREZ (2004). "Caracterización del sistema de innovación para el Distrito Capital”; dir. José Javier Aguilar Zambrano. Tesis de Maestría en Ciencias Económicas. Bogotá: Universidad Nacional de Colombia.

-PORTER MICHAEL, E. (2000). Estrategia competitiva: técnicas para el análisis de los sectores industriales y de la competencia. México: Editorial Continental.

-RESTREPO, María C. y ROSERO, Xiomara (2002). "Teoría institucional y proceso de internacionalización de las empresas colombianas". Estudios Gerenciales. Bogotá: Universidad ICESI, julio-septiembre, No 84.

-SENA (2003). "Orientaciones para la coordinación y seguimiento en la transferencia de tecnología de los proyectos beneficiados en el Programa Nacional de Apoyo y Fortalecimiento de Incubadoras de Empresas de Base Tecnológica”. Dirección General. Documento de trabajo - versión $\mathrm{N}^{\circ} .3$ http://www.sena.edu.co/downloads/PLAN_ESTRATEGICO_Nov_1403.pdf 4/5/05.

-TRUJILLO, M. A., RODRÍGUEZ, D. F., GUZMÁN, A., BECERRA, G. (2004). "Perspectivas teóricas sobre la internacionalización de empresas”. Cuadernos de investigación. Bogotá: Universidad del Rosario. Facultad de Administración de Empresas. No 3.

-URIBE, Elsa M. (2001). "Aportes en la dirección por competencias básicas distintivas a la explicación de las operaciones internacionales". Cuadernos de Administración. Bogotá: Pontificia Universidad Javeriana. $\mathrm{N}^{\mathrm{o}} 14$, junio.

-URIBE, Elsa M. (1998)."Las formas de entrada a los mercados extranjeros: base para el diseño de las estrategias de internacionalización empresarial". Cuadernos de Administración. Bogotá: Pontificia Universidad Javeriana. No 21. 